\title{
Analyst
}

Check for updates

Cite this: Analyst, 2022, 147, 534

Received 4th November 2021

Accepted 11th January 2022

DOI: $10.1039 /$ d1an02005f

rsc.li/analyst

\section{Fluoroponytailed ionic liquids as co-porogens for poly(butyl methacrylate-co-ethylene dimethacrylate) monolithic supports for thin layer chromatography $\dagger$}

\author{
Manuel Otter, ${ }^{a}$ Gabriel Partl, (D) ${ }^{\mathrm{b}}$ Michael Noisternig ${ }^{\mathrm{c}}$ and Rania Bakry (D) *a \\ Porous layered monolithic substrates of poly(butyl methacrylate-co-ethylene dimethacrylate) were syn- \\ thesized via UV initiated free radical polymerization in the presence of fluoroponytailed ionic liquids as \\ co-porogenic constituents. The effects of the type and the amount of selected fluorous ionic liquids on \\ various properties of the monolithic support, e.g. porosity, specific surface area and chromatographic \\ performance, in particular for their usability in reversed phase TLC, were examined. Porosity was charac- \\ terized by means of mercury porosimetry and scanning electron microscopy. The monolithic stationary \\ phases with different layer thickness were successfully applied in the separation of three curcuminoids, \\ namely curcumin, demethoxycurcumin and bisdemethoxycurcumin. Relative retention factor, theoretical \\ plates and resolution were used for the evaluation of the monolithic support's performance. To verify the \\ feasibility of the monoliths, the method was employed for the discrimination between the plant species \\ Curcuma longa and Curcuma xanthorrhiza.
}

\section{Introduction}

Over the past decades, porous polymeric monoliths sustained remarkable progress, in terms of stationary phase development for chromatographic separations. The ease of preparation, tuneable porous structure, available surface chemistry and task specific tailoring expanded their scope of applications. ${ }^{1,2}$ Several parameters can influence the porous structure including porogens, monomer composition, polymerization time, initiator type and reaction temperature. ${ }^{3}$

Porogens, pore generating solvents, are the most important components of the polymerization mixture affecting the surface area, porosity and morphology of the resulting monolithic structure. The porogens used for the preparation of porous monoliths by in situ free radical polymerization mostly consist of a mixture of solvating and non-solvating diluents. Solvating porogens are mostly responsible for the generation

\footnotetext{
${ }^{a}$ Institute of Analytical Chemistry and Radiochemistry, Leopold-Franzens-University Innsbruck, Innrain 80-82, 6020 Innsbruck, Austria. E-mail: rania.bakry@uibk.ac.at; Fax: +43 512507 57399; Tel: +43 51250757308

${ }^{b}$ Institute of General, Inorganic and Theoretical Chemistry, Leopold-FranzensUniversity Innsbruck, Innrain 80-82, A-6020 Innsbruck, Austria

${ }^{c}$ Institute of Pharmacy, Pharmaceutical Technology, Leopold-Franzens-University Innsbruck, Innrain 52c, A-6020 Innsbruck, Austria

$\dagger$ Electronic supplementary information (ESI) available. See DOI: 10.1039/ d1an02005f
}

of micro-/mesopores, whereas non-solvating types are attributed to generate macropores. ${ }^{4}$ The choice of porogen depends on the polarity and solubility of monomers, polymer and coporogens. Owing to the different characteristics of porogens, they generate different pore architectures within the polymeric structure. The solvating porogens are miscible with the monomers as well as the initially formed oligomers, resulting in late-stage phase separation at higher conversion of monomer to polymer. As a result, a scaffold with interconnected individual microglobules is formed. Conversely, non-solvating porogens are responsible for early-stage phase separation at lower conversion, due to their poor miscibility in the polymerization system. This process causes the aggregation of the microglobules and the formation of a scaffold with low surface area.

Organic solvents and organic polymers are commonly used porogens. ${ }^{5}$ The main factors governing the choice of suitable porogens are their molecular size, alkyl chain length and solubility profiles. ${ }^{6}$ Most organic liquids can be used in monolith fabrication, even volatile solvents can be employed in the preparation through photoinitiated polymerization. During the last decade, ionic liquids (ILs) attracted growing attention as porogens or co-porogens. ${ }^{7-9}$ The most widely used porogen ILs are 1-butyl-3-methylimidazolium salts, in particular with $\mathrm{BF}_{4}{ }^{-}$, $\mathrm{PF}_{6}{ }^{-}, \mathrm{HSO}_{4}{ }^{-}$anions. ${ }^{5}$ They offer numerous advantages, including negligible volatility as well as highly tuneable solubility profiles. ILs may even significantly enhance the polymerization rate, through acceleration of the rate of monomer propa- 
gation. ${ }^{10}$ Expectedly, the presence of ILs also affects the morphology of the monolith by altering swelling and shrinking behaviour of the monolith. However, the benefit of ILs on the chromatographic separations in thin layer format is not reported.

In our previous work, we demonstrated the thin layer chromatographic separation of peptides and proteins on poly(butyl methacrylate-co-ethylene dimethacrylate) (poly(BMA-EDMA)) monoliths followed by MALDI-TOF MS detection. The monoliths were prepared by photoinitiated free radical polymerization using dodecanol and cyclohexanol as porogens. ${ }^{11}$ The resulting monoliths were successfully used for the separation of peptides and proteins. However, their application for the separation of small molecules was not satisfactory. Svec's group improved the efficiency for the separations of small molecules by hypercrosslinking poly(4-methylstyrene-cochloromethylstyrene-co-divinylbenzene) via Friedel-Crafts alkylation. ${ }^{12}$ Maksimova et al., studied the separation low molecular weight dyes and dinitrophenol amino acids using a series of methacrylate based monolithic support. Several functional monomers were used including glycidyl methacrylate, butyl methacrylate, aminoethyl methacrylate, 2-hydroxyethyl methacrylate and 2-cyanoethyl methacrylate. ${ }^{13}$ Later on, Yin et al. demonstrated the application of macroporous poly HIPE monolithic layers based on poly(styrene-butyl acrylate-divinylbenzene) for the identification of Chinese herbal medicinal components. ${ }^{14}$ Recently, Korzhikova-Vlakh et al. summarizes the current achievement on the application of microporous polymer monolithic layers for thin layer chromatography. ${ }^{15}$

In this work, we focused on the development of planar monoliths with optimized porosity for the separation of small molecules based on poly(BMA-EDMA) using specific ILs, mainly fluoroponytailed room temperature ILs (FILs), as coporogens. The selected FILs were synthesized using efficient and cost-effective procedures developed by Schottenberger's group. ${ }^{16,17}$ The monolithic supports were prepared using ternary porogenic solvents consisting of cyclohexanol, 1-dodecanol or 1-decanol, and 2-(1H,1H,2H,2H-perfluorooctylthio)-1methylimidazolium with varying counter ions (Fig. 1). The effect of FILs concentration on the porous structure of monolithic support was studied. The resulting monoliths were morphologically characterized by scanning electron microscopy and mercury porosimetry. The performance of the developed monoliths was assessed for their suitability to separate three curcuminoids by thin layer chromatography. Furthermore, the monolithic plates were applied for discrimination between two Curcuma species.

\section{Materials and methods}

\subsection{Materials}

Butyl methacrylate (BMA) (99\%, containing hydroquinone monomethyl ether (MEHQ) as inhibitor); ethylene glycol dimethacrylate (EDMA) (98\%, containing 90-110 ppm MEHQ as inhibitor); 2,2'-dimethoxy-2-phenylacetophenone (DMPA)
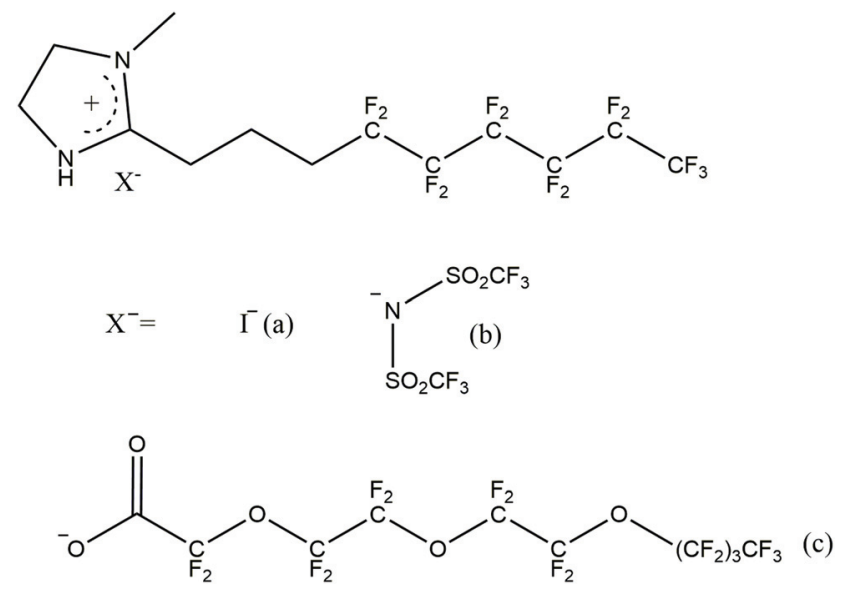

Fig. 1 Chemical structure of the utilized FILs, $2-(1 H, 1 H, 2 H, 2 H$-perfluorooctylthio)-1-methylimidazolium: iodide (a), triflimide (b), perfluoro3,6,9-trioxatridecanoate (c).

(99\%); 1-decanol (98\%); 1-dodecanol (98\%); cyclohexanol 99\%; acetic acid (96\%); ethanol (EtOH) (96\%); 3-(trimethoxysilyl) propyl methacrylate (98\%); curcumin (99.5\%); curcuminoids ( $\geq 94 \%$ curcuminoid content, $\geq 80 \%$ curcumin), methyl red (indicator ACS, Reag. Ph Eur), methylene blue (Reag. Ph Eur) and 2,5-dihydroxybenzoic acid (DHB) (puriss. p.a) were purchased from Sigma Aldrich (Vienna, Austria). Acrylate monomers were passed through basic Aluminium oxide packed cartridges to remove inhibitors. Chloroform (anhydrous, contains amylene as stabilizer, $\geq 99 \%$ ) (Attention: carcinogenic); dichloromethane (anhydrous, $\geq 99.8 \%$, contains 40-150 ppm amylene as stabilizer); HPLC grade acetonitrile (ACN) and trifluoroacetic acid (TFA) ( $\geq 99.5)$; were purchased from Merck (Vienna, Austria). Five samples of curcuma species i.e. Curcuma longa (3 samples) and Curcuma xanthorrhiza (2 samples) were collected from different areas in the middle of Java, Indonesia. Water was purified by a Milli-Q unit (Merck Millipore, Darmstadt, Germany) and was used for all experiments. Glass plates $76 \times 26 \times 1 \mathrm{~mm}$ form MEnzel-Glaeser (Braunschweig, Germany). Photoinitiated polymerization was performed using an UV Crosslinker CX-2000 UVP (Jena, Germany).

\subsection{FILs synthesis}

The selected FILs (Fig. 1) were prepared as described by M. Hummel et al. ${ }^{17}$ Briefly, $100.0 \mathrm{~g}$ of 1-methyl-1,3-dihydro- $2 \mathrm{H}^{-}$ imidazole-2-thione (methimazole) and 500.0 $\mathrm{g}$ of $1 \mathrm{H}, 1 \mathrm{H}, 2 \mathrm{H}, 2 \mathrm{H}$-perfluorooctyl iodide were dissolved in $500 \mathrm{ml}$ of ethanol under gentle heating $\left(\approx 45{ }^{\circ} \mathrm{C}\right)$. Following complete dissolution, the reaction mixture was refluxed for $36 \mathrm{~h}$; after cooling, the solution was concentrated to about half of its initial volume. To this warm saturated solution, $700 \mathrm{ml}$ of diethyl ether was added quickly under stirring, which caused the product to precipitate as a yellowish, chunky solid. The resulting mixture was shaken for several minutes, after which the product was filtered off and washed with another $700 \mathrm{ml}$ 
of diethyl ether. Finally, the obtained white, waxy product was dried in high vacuum overnight. Afterwards, the counter ion metathesis of the fluoroponytailed methimazolium iodide was carried out to obtain the bis(trifluoromethanesulfonyl)imide (triflimide) and perfluoro-3,6,9-trioxatridecanoate salts, respectively.

\subsection{Preparation of the monolithic layer}

The synthesis of the monolith was performed in two steps using our previously developed procedures. ${ }^{11}$ In the first step, the surfaces of the glass supports were modified in order to enable the covalent attachment of the monolith. Glass plates were washed with acetone and water and treated with $1 \mathrm{M}$ sodium hydroxide for $30 \mathrm{~min}$, washed with water and subsequently with $1 \mathrm{M} \mathrm{HCl}$ for $30 \mathrm{~min}$, then finally rinsed with water and acetone. The plates were dried at $65{ }^{\circ} \mathrm{C}$ for $1 \mathrm{~h}$. Afterwards, the surfaces were treated using a $20 \%$ solution of 3 -(trimethoxysilyl)propyl methacrylate in $95 \%$ ethanol, adjusted to pH 5 using 96\% acetic acid for $2 \mathrm{~h}$ at room temperature. The modified plates were washed with ethanol and dried at $65^{\circ} \mathrm{C}$ for $12 \mathrm{~h}$. The monomer, crosslinker, porogenic solvents and photoinitator (DMPA) were mixed in a glass vial and ultrasonicated for $10 \mathrm{~min}$. The detailed compositions of the polymerization mixtures are listed in Table 1 . The polymerization mixtures were poured onto a rectangular mould consisting of two treated glass plates and a Teflon gasket with thickness ranging from $25-200 \mu \mathrm{m}$ and clamped. The mould assembly was exposed to UV light at $254 \mathrm{~nm}$ for $30 \mathrm{~min}$. After completion of polymerization, the mould was disassembled. The glass plate, with firmly attached monolithic layer, was immersed in EtOH overnight to remove the porogens, and then the plates were dried at $60^{\circ} \mathrm{C}$ for $2 \mathrm{~h}$.

\subsection{Samples and matrix solutions}

Curcuminoids standard solutions were prepared in $80 \mathrm{vol} \%$ EtOH $\left(1 \mathrm{mg} \mathrm{ml}^{-1}\right)$. The stock solutions were further diluted as required. Methylene blue and methyl red solutions with a concentration of $0.3 \mathrm{mg} \mathrm{ml}^{-1}$ were prepared by dissolution in 80 vol\% EtOH. A solution of $20 \mathrm{mg} \mathrm{ml} \mathrm{m}^{-1}$ of DHB in $1: 1$ ACN : $\mathrm{H}_{2} \mathrm{O}$, containing $0.1 \%$ TFA, was prepared. $100 \mathrm{mg}$ of curcuma powder was extracted with $50 \mathrm{ml}$ of EtOH $80 \mathrm{vol} \%$ by ultrasonication for $10 \mathrm{~min}$, followed by filtration. The filtrates were diluted as required.

\subsection{TLC separation of curcuminoids}

A sample $(0.5 \mu \mathrm{L})$ was spotted onto the monolithic plate and $1 \%$ TFA in chloroform was used as mobile phase. The developing tank was conditioned for $30 \mathrm{~min}$ before plate development. After development the plates were air-dried and visualized by UV illumination at $366 \mathrm{~nm}$. For MALDI analysis the spots were scratched off, transferred to $1.5 \mathrm{ml}$ Eppendorf vials and extracted with methanol. MALDI-TOF MS measurements were carried out using an Ultraflex I MALDI-TOF/TOF instrument (Bruker Daltonics). For the acquisition of the mass spectra, 100 laser shots were typically applied at several positions. Only positively charged compounds were analyzed, and 500 singleshot spectra were accumulated to obtain an appropriate signalto-noise ratio. Spectra were recorded in reflector mode. The Flex Analysis version 2.4 software packages provided by the manufacturer were used for data processing.

\subsection{Characterization methods}

SEM images of the monolith were recorded on a Jeol JSM-6010LV instrument (Tokyo, Japan) by applying an acceleration voltage of $10 \mathrm{kV}$. The monolithic polymer was scraped off the glass support, and then dried under vacuum. Then a small part of the monolith sprinkled with gold for SEM. Mercury intrusion porosimetry was used to evaluate the pore size distribution and specific surface area of the polymeric monolith, therefore a Pascal 140 (Thermo Fisher Scientific, I-Milano) and a Porosimeter 2000 (Carlo Erba, I-Milano) were applied. The FT-IR method was used to confirm the chemical composition of the monolith and that the FILs are not incorporated in the polymeric structure. Before measurement, the monolith was scraped off and washed several times with ACN then dried for $24 \mathrm{~h}$. The spectra were obtained with a Bruker ALPHA-P FT spectrometer in ATR mode.

\subsection{Chromatographic calculations}

Retardation factor $\left(R_{\mathrm{f}}\right)$, resolution $\left(R_{\mathrm{S}}\right)$, and number of theoretical plates were used to evaluate the

Table 1 Composition of monolithic plates

\begin{tabular}{|c|c|c|c|c|c|c|}
\hline Monolith & $\frac{\text { Monomer }}{\operatorname{BMA}(\% \mathrm{w} / \mathrm{w})}$ & $\frac{\text { Crosslinker }}{\operatorname{EDMA}(\% \mathrm{w} / \mathrm{w})}$ & \multicolumn{4}{|l|}{ Porogenic solvent } \\
\hline III & 24.0 & 16.0 & 40.0 & & 10.0 & $10.0^{b}$ \\
\hline IV & 24.0 & 16.0 & 40.0 & & 10.0 & $10.0^{c}$ \\
\hline V & 18.0 & 12.0 & 46.7 & & 11.7 & $11.6^{a}$ \\
\hline
\end{tabular}

${ }^{a}$ Iodide. ${ }^{b}$ Triflimide. ${ }^{c}$ Perfluoro-3,6,9-trioxatridecanoate. 
chromatographic performance of the thin layer plates according to equations: ${ }^{18}$

$$
\begin{gathered}
R_{\mathrm{f}}=\frac{L}{M} \\
R_{\mathrm{S}}=2 \frac{R_{\mathrm{f} 2}-R_{\mathrm{f} 1}}{W_{1}+W_{2}} \\
N=16 \frac{L \cdot M}{W^{2}}
\end{gathered}
$$

where $M$ and $L$ are the migration lengths of the mobile phase and analyte, and $W$ is the peak or spot width of the analyte.

\section{Results and discussion}

\subsection{Preparation and characterization of the monolithic plates}

Utilizing FILs as co-porogens, a set of poly(BMA-EDMA) monolithic plates were prepared according to the conditions listed in Table 1 under an irradiation wavelength of $254 \mathrm{~nm}$ for $30 \mathrm{~min}$.

As mentioned introductorily, the effect of FILs on the pore morphology of the monolithic layers can vividly be elucidated by microscopy. Fig. 2I shows the SEM images of porous structure of monoliths with conventional (generic) composition exhibiting its typical porous features (monolith I). However, the monoliths (II-IV) prepared using $10 \% \mathrm{w} / \mathrm{w}$ FILs as coporogen with $40 \% \mathrm{w} / \mathrm{w}$ 1-decanol and $10 \%$ cyclohexanol exhibit denser porous structures (Fig. 2II-IV). The denser porous structure can be attributed to the good solvating properties of the FILs towards the monomers. Therefore, the local concentration of monomers in FILs is higher than that in the precipitated, insoluble "gel-like" species (nuclei), leading to individualized small globules. Additionally, the large number of nuclei competing for the remaining monomers results in high numbers of small globules that aggregate with small pores, as can be seen in Fig. 2II-IV. ${ }^{19}$ Additionally, it can be noticed that changing the anion of the FILs has a remarkable effect on pore architecture and polymeric backbone density. The use of the triflimide FIL causes a non-uniform monolithic structure, in contrast to the monoliths prepared utilizing iodide and perfluoro-3,6,9-trioxatridecanoate FILs. However, the flow channels are wider with triflimide and perfluoro3,6,9-trioxatridecanoate (monolith III and IV), compared to monolith II prepared with iodide. Furthermore, decreasing the ratio of monomer to porogen from $40 \%$ to $30 \%$, as is the case for monolith $\mathrm{V}$, resulted in wider flow channels with larger globule size (Fig. 2V). On the other hand, the incorporation of 1-dodecanol instead of 1-decanol (monolith VI) into the monomeric mixture effected a remarkable change in morphology (Fig. 2II and VI). 1-Dodecanol has less solvating power for the polymer chains in comparison to 1-decanol, resulting in earlier phase separation. As can be noticed from Fig. 2VI, the porous scaffold is less dense and possesses larger globule sizes with homogenous through-pores and polymer clusters, in comparison to monolith II. Consequently, the change in morphology was reflected in the changes in the specific surface area of the monoliths. The utilization of the FILs as co-porogens resulted in approximately $100 \%$ increase in specific surface area, since the nuclei retain their individuality. Monolith VII, prepared without FILs, possesses a surface area of $16 \mathrm{~m}^{2} \mathrm{~g}^{-1}$. Conversely, the use of $10 \%$ FILs resulted in

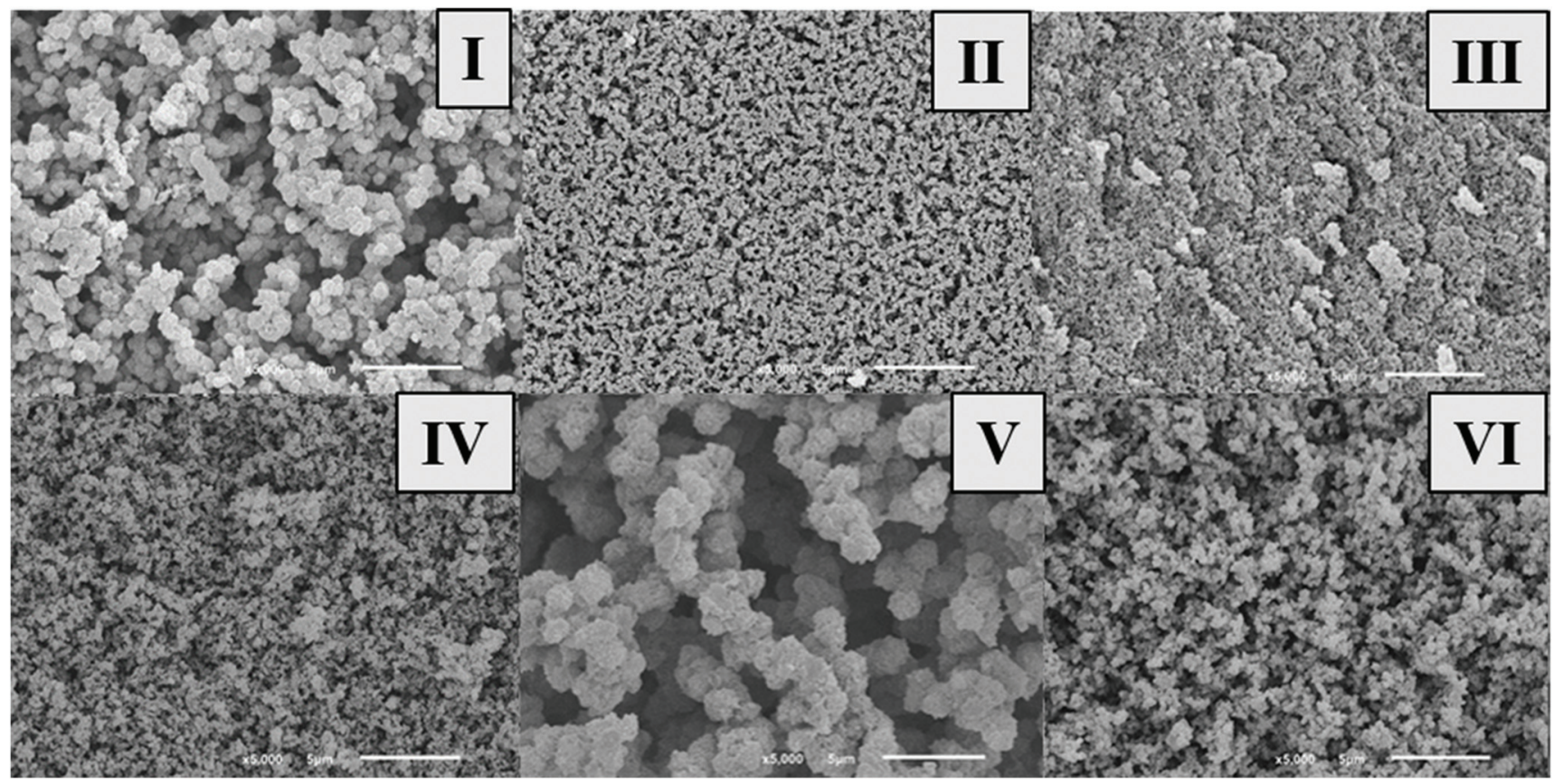

Fig. 2 Scanning electron microscope images of monoliths (VI and VII) prepared according to composition summarized in Table 1. 


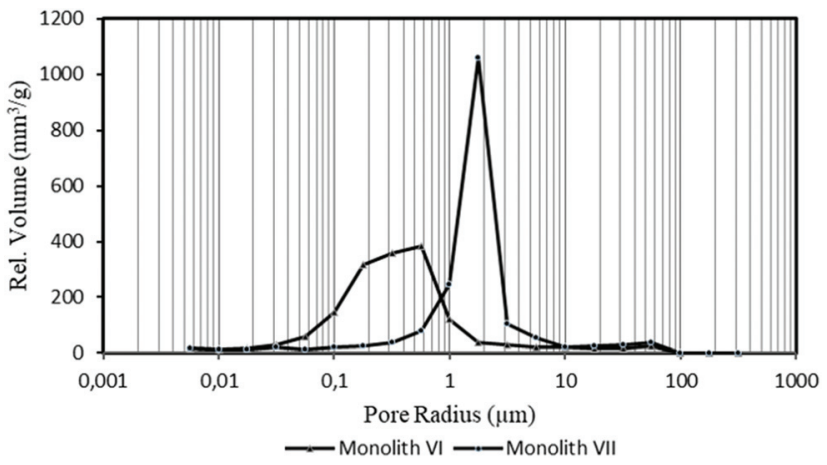

Fig. 3 Pore size distribution of poly(BMA-EDMA) monoliths VI (with $10 \%$ FIL-iodide) and VII (without FIL).

monoliths with surface areas up to $33 \mathrm{~m}^{2} \mathrm{~g}^{-1}$ (monolith VI). This increase in the surface area can be attributed to the increase of clusters with smaller size. As mentioned before, the presence of the ILs accelerates the polymerization rates accompanied with late phase separation, which would produce microglobules. ${ }^{10}$

Furthermore, the influence of the incorporation of FILs as co-porogens on the pore size distribution of the developed monolithic supports was examined using mercury porosimetry. Pore size distribution indicates the accessible fraction of total pore volume. As can be shown in Fig. 3, both monolithic plates VI and VII possess monomodal pore size distribution. Monolith VI, prepared using 10\% iodide FIL, 1-dodecanol and cyclohexanol as porogens, displays a comparatively broad pore size distribution profile with total intrusion volume of $1.62 \mathrm{ml}$ $\mathrm{g}^{-1}$ and average pore radius of $0.360 \mu \mathrm{m}$, resulting in a total porosity of $63.0 \%$. However, monolith VII, prepared without FILs, exhibits a pore size distribution with total intrusion volume of $1.81 \mathrm{ml} \mathrm{g}^{-1}$ at a pore radius of $1.5 \mu \mathrm{m}$, with a total porosity of $65.7 \%$ (Fig. 3).

FT-IR was utilized to confirm that the FILs were not incorporated into the formed monolith. The FT-IR spectrum of e.g. monolith VI did not show any evidence towards the presence of any FIL-specific IR bands. The spectra of plates I and VI are identical in regard to the polymer's characteristic absorption bands (ESI $1 \uparrow)$.

\subsection{Permeability of the monolithic plates and velocity constant of the mobile phase}

Permeability of the monolithic plates is a decisive property for their application as separation media. The migration of the mobile phase is controlled by capillary forces, which is due to the decrease in free energy of the solvent as it goes through the porous structure of the monolithic layer. ${ }^{20}$ Migration of the mobile phase is a function of the distance between the front and the solvent level in the developing chamber. The porous stationary phase can be described as a bunch of very fine capillaries where the mobile phase more likely coheres rather than adheres to the capillary wall. This capillary flow is dependent on the characteristics of the stationary phase and the viscosity and surface tension of the selected mobile phase. ${ }^{21}$ It can be determined from the time required for the mobile phase to move between fixed points marked on the TLC plate. The velocity constant can be calculated according to the following equation: ${ }^{22,23}$

$$
\left(Z_{\mathrm{f}}\right)^{2}=k t
$$

where $Z_{\mathrm{f}}$ is the distance between the solvent front and the solvent source, $t$ is the required time and $k$ is the velocity constant.

Table 2 summarizes the velocity constant of the developed monoliths with $150 \mu \mathrm{m}$ thickness using $\mathrm{ACN} / \mathrm{H}_{2} \mathrm{O}(60 / 40)$ with $0.1 \%$ TFA. The plates are used for reasonable distances ranging from $30-60 \mathrm{~mm}$. Monolith I exhibits a velocity constant of approximately $2.67 \mathrm{~mm}^{2} \mathrm{~s}^{-1}$, whereas monoliths II-IV show velocity constants ranging from 0.49 to $1.30 \mathrm{~mm}^{2} \mathrm{~s}^{-1}$. The results are following SEM images, whereas monolith I demonstrates wider flow-channel compared to monoliths IIIV. Monolith V, prepared with only $30 \%$ monomer content, possesses a highly porous scaffold and shows the highest permeability with a velocity constant of $6.08 \mathrm{~mm}^{2} \mathrm{~s}^{-1}$.

\subsection{Chromatographic performance of the developed monolith}

Complementarily, the chromatographic behaviour of the monolithic plates was examined using dye mixtures of methylene blue and methyl red, and applying a mobile phase consisting of a $60 / 40 \mathrm{v} / \mathrm{v} \mathrm{ACN} / \mathrm{H}_{2} \mathrm{O}$ mixture containing 0.1\%TFA. The impact of polymer composition on the separation of the two dyes is summarized in Table 2. The separation can be achieved on all polymeric plates with a resolution ranging from 0.29 and 1.2. The resolution $R_{\mathrm{S}}$ is a practised criterion of the separation degree to two analytes and $R_{\mathrm{S}}>1.0$ is necessary for a basic separation and $R_{\mathrm{S}}>1.5$ for baseline separation. As shown in Table 2, monolith VI presents better resolution of 1.8 with reduced spot width of 0.10 and $0.20 \mathrm{~mm}$ for methylene blue and methyl red respectively, in comparison to other monoliths. Therefore, for all subsequent experiments monolith VI was chosen in order to obtain high performance.

Furthermore, to evaluate the feasibility of the developed plates for the separation of small molecules, curcumin (CurI), demethoxycurcumin (CurII) and bisdemethoxycurcumin

Table 2 Chromatographic parameters of the monoliths using 60/40 $\mathrm{v} / \mathrm{v} \mathrm{ACN} / \mathrm{H}_{2} \mathrm{O}$ mixture containing $0.1 \%$ TFA as mobile phase

\begin{tabular}{|c|c|c|c|c|c|c|}
\hline \multirow[b]{2}{*}{ Monolith } & \multicolumn{2}{|c|}{ Methylene blue } & \multicolumn{2}{|c|}{ Methyl red } & \multirow[b]{2}{*}{$R_{\mathrm{S}}$} & \multirow[b]{2}{*}{$\begin{array}{l}\text { Velocity } \\
\text { constant } \mathrm{mm}^{2} \\
\mathrm{~s}^{-1}\end{array}$} \\
\hline & $R_{\mathrm{f}}$ & $\begin{array}{l}\text { Spot } \\
\text { width } \\
\text { mm }\end{array}$ & $R_{\mathrm{f}}$ & $\begin{array}{l}\text { Spot } \\
\text { width } \\
\mathrm{mm}\end{array}$ & & \\
\hline I & 0.88 & 0.2 & 0.77 & 0.35 & 0.40 & 2.66 \\
\hline II & 0.66 & 0.4 & 0.32 & 0.55 & 0.72 & 0.49 \\
\hline III & 0.87 & 0.6 & 0.70 & 0.55 & 0.29 & 0.83 \\
\hline IV & 0.93 & 0.5 & 0.58 & 0.5 & 0.70 & 1.29 \\
\hline V & 0.89 & 0.18 & 0.65 & 0.3 & 1.13 & 6.08 \\
\hline VI & 0.87 & 0.2 & 0.60 & 0.2 & 1.20 & 2.01 \\
\hline
\end{tabular}


(CurIII) are selected as model analytes (ESI $\dagger \dagger$ ). Firstly, the mobile phase applied for chromatographic separation was optimized. Different mobile phase compositions were tested, using monoliths VI and VII, namely $\mathrm{ACN} / \mathrm{H}_{2} \mathrm{O} 60 / 40, \mathrm{CH}_{2} \mathrm{Cl}_{2}$ and $\mathrm{CHCl}_{3}$ to obtain good resolution and reproducible separation. With $\mathrm{ACN} / \mathrm{H}_{2} \mathrm{O}$, no separation could be achieved on both monoliths. Using $\mathrm{CH}_{2} \mathrm{Cl}_{2}$ and $\mathrm{CHCl}_{3}$, the three curcuminoids could not be separated on monolith VII, prepared without FILs. A yellow smear was obtained for CurI und CurII. However, employing monolith VI good separation could be

Table 3 Chromatographic performance of poly(BMA-EDMA) monolithic plate (monolith $\mathrm{VI}$ ), with $150 \mu \mathrm{m}$ thickness, using curcuminoids, applying $\mathrm{CHCl}_{2}$ and $\mathrm{CHCl}_{3}$ as mobile phases

\begin{tabular}{lllll}
\hline $\begin{array}{l}\text { Mobile phase } \\
\text { Analyte }\end{array}$ & $R_{\mathrm{f}}( \pm \mathrm{SD})$ & Spot width $(\mathrm{mm})$ & $N$ & $R_{\mathrm{S}}$ \\
\hline CHCl $_{2}$ & & & & \\
CurI & $0.18 \pm 0.006$ & 3.2 & 336.91 & 5.67 \\
CurII & $0.67 \pm 0.137$ & 2.6 & 1878.10 & 1.41 \\
CurIII & $0.88 \pm 0.002$ & 6.7 & 375.05 & \\
& & & & \\
CHCl $_{3}$ & & & 209.02 & 5.85 \\
CurI & $0.17 \pm 0.048$ & 2.4 & 1666.44 & 3.33 \\
CurII & $0,65 \pm 0.044$ & 1.7 & 2539.69 & \\
CurIII & $0.87 \pm 0.036$ & 1.6 & &
\end{tabular}

obtained, with $\mathrm{CHCl}_{3}$ giving superior results in terms of resolution and spot width. These results showed that, the incorporation of FILs in monolith synthesis improved notably of the separation efficiency of the monolithic plates.

Table 3 summarizes the data obtained by using $\mathrm{CHCl}_{3}$ and $\mathrm{CH}_{2} \mathrm{Cl}_{2}$ as mobile phases on monolith VI. For example, the spot width for curcumin using $\mathrm{CHCl}_{3}$ was $1.7 \mathrm{~mm}$, whereas with $\mathrm{CH}_{2} \mathrm{Cl}_{2}$ it was $6.7 \mathrm{~mm}$. The plates are used for distances and speeds ranging from 3-6 cm in 8-9 min, since longer distances resulting in impaired separation because of diffusion in the layer. The highest number of theoretical plates, namely 2539.69, was obtained with CurI using $\mathrm{CHCl}_{3}$ as mobile phase.

Fig. 4 demonstrates a representative separation of curcuminoids standard solution on monolith VI using $\mathrm{CHCl}_{3}$ as mobile phase. The plates were visualized using UV light with a wavelength of $365 \mathrm{~nm}$. However, since clear identification of spots is not possible by visualization or staining, the spots were scraped off the TLC plate and extracted with methanol. The extracted curcuminoids were loaded on the MALDI target combined with DHB matrix. MALDI-MS offers a soft ionization technique for a fast and simple method for identification of the separated spots. MALDI-MS spectra of the 3 curcuminoids shown in Fig. 4(b-d) indicate the good separation behaviour of

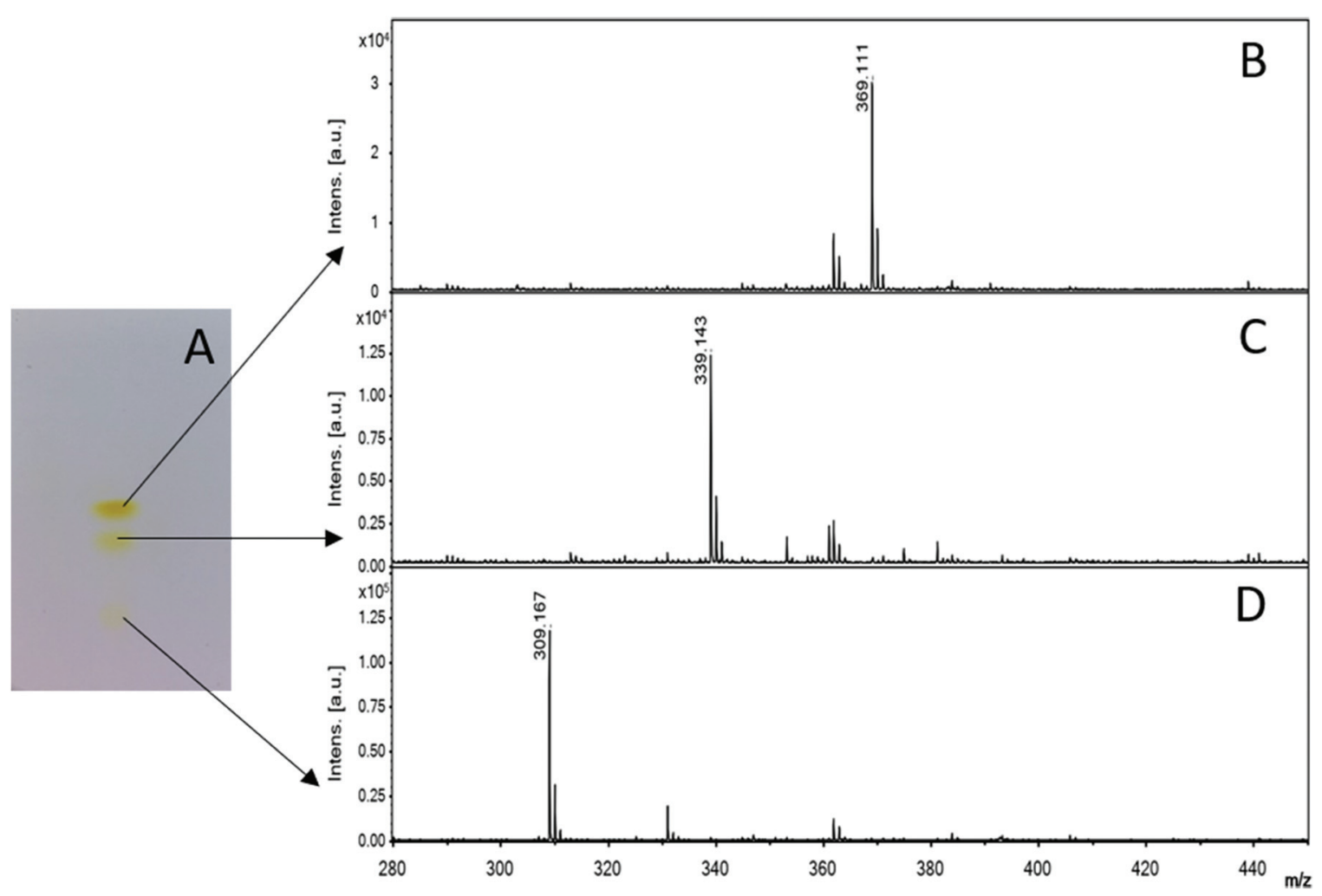

Fig. 4 TLC separation of curcuminoids mixture on $150 \mu \mathrm{m}$ thick poly(BMA-EDMA) monolithic layer (monolith VI) attached to a glass plate using $\mathrm{CHCl}_{3}$ as mobile phase; sample volume $1.0 \mu \mathrm{L}$, viewed under UV $365 \mathrm{~nm}$ (A). MALDI-TOF MS spectra of extracted spots of CurlII (B), Curll (C), and Curl (D) using DHB as matrix. 
the monolithic plates. As can exemplified by Fig. 4 b, the $m / z$ of 369 Da clearly corresponds to the $[\mathrm{M}+\mathrm{H}]^{+}$-peak of CurI. In Fig. $4 \mathrm{c}$, the $\mathrm{m} / \mathrm{z}$ of 339 Da correlates to CurII, and the $\mathrm{m} / \mathrm{z}$ of 309 Da corresponds to CurIII (Fig. 4d). Coupling of TLC and MALDI-MS is one of the promising hybrid analytical methods. It opens broad possibilities regarding identification, screening, methods development for the analysis of wide range of analytes. $^{24}$

The effect of monolithic layer thickness on the chromatographic separations of curcuminoids was investigated. Several monolithic plates were prepared with a thickness between 25 and $200 \mu \mathrm{m}$. Using monolithic plates with a thickness of 25 and $50 \mu \mathrm{m}$, no separation of the three curcuminoids could be obtained due to the low capacity of the monolith. However, the good separation was obtained using a monolithic layer with $150 \mu \mathrm{m}$ thickness. Additionally, the reusability of the monolithic plates was examined by repeated use after washing with ethanol and drying at $60^{\circ} \mathrm{C}$. A reproducible separation of the three curcuminoids was achievable even after five times.

\subsection{Discrimination between Curcuma longa and Curcuma xanthorrhiza}

Conclusively, the monolithic plates were applied to differentiate between Curcuma longa and curcuma xanthorrhiza, based on the presence of CurI, CurII, and CurIII (Fig. 5). Four samples from different regions of Indonesia were pulverized, extracted with $80 \%$ EtOH and loaded onto the monolithic plates. Fig. 5A illustrates the plates with Curcuma longa (C.L.1 and C.L.2) exhibiting fluorescence spots of CurI, CurII, and CurIII, with $R_{\mathrm{f}}$ of $0.121,0.623$ and 0.848 respectively. The separation was accomplished in $7 \mathrm{~min}$. In case of curcuma xanthorrhiza (C.X.1 and C.X.2) (Fig. 5B) only two curcuminoids could be detected namely CurI and CurII, with $R_{\mathrm{f}}$ of 0.77 and 0.81 , respectively (Table 4). From these results, it is evident that by using the monolithic plates it was possible to qualitatively discriminate between the two species of curcuma in a fast and simple manner. The separation employing the developed plates is in accordance with the results previously published using commercially available silica TLC plates. ${ }^{25,26}$
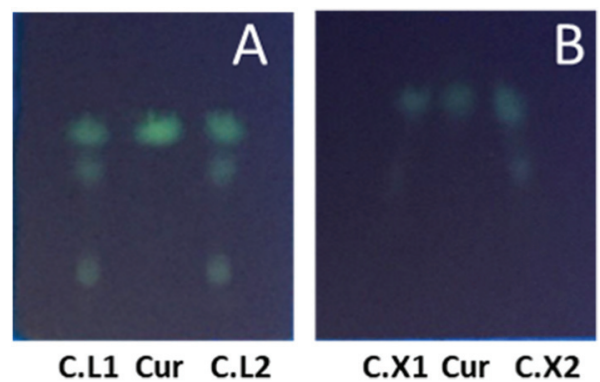

Fig. 5 TLC images of the separation of Curcuma longa (A) and curcuma xanthorrhiza (B) roots extract on $150 \mu \mathrm{m}$ thick poly(BMA-EDMA) monolithic layer (monolith $\mathrm{VI}$ ) using $\mathrm{CHCl}_{3}$ as mobile phase, viewed under $365 \mathrm{~nm}$ UV lamp.
Table 4 Chromatographic performance of Curcuma roots extract on monolith $\mathrm{VI}$, applying $\mathrm{CHCl}_{3}$ as mobile phase

\begin{tabular}{lllll}
\hline Plant & Origin & CurI $( \pm$ SD $)$ & CurII $( \pm$ SD $)$ & CurIII $( \pm$ SD $)$ \\
\hline Cur.L.1 & Sleman & $0.12 \pm 0.032$ & $0.62 \pm 0.093$ & $0.85 \pm 0.014$ \\
Cur.L.2 & Playen & $0.21 \pm 0.001$ & $0.66 \pm 0.045$ & $0.90 \pm 0.001$ \\
Cur.Xan.1 & Naggulan & & $0.65 \pm 0.035$ & $0.85 \pm 0.042$ \\
Cur.Xan.2 & Girimulyo & & $0.67 \pm 0.020$ & $0.85 \pm 0.060$
\end{tabular}

Cur.L.: Curcuma Lunga; Cur.Xan.: Curcuma Xanthorriza; curI: bisdemethoxycurcumin; CurII: demethoxycurcumin; CurIII: curcumin.

\section{Concluding remarks}

Porous polymers with controlled porous properties have been successfully produced by photoinitiated free radical polymerization under additional utilisation of FILs. The porosity of the resulting polymers is mainly dependent on the concentration of porogens. While the conventional poly(BMA-EDMA) monolith could be successfully used for the separation of peptides and proteins, its application for the separation of small molecules was not satisfactory. Therefore, we altered the developed plates using FILs as co-porogens for the separation of small molecules. The utilization of FILs resulted in reduced permeability and formation of denser porous scaffolds in comparison to the monoliths prepared in absence of FILs. The positive effect of FILs on the separation performance of the monolithic plates could be noticed in the separation of curcuminoids and is comparable with separation using conventional thin layer plates. The novel FIL-modified plates illustrated their potential in the differentiation of closely related medicinal plants for identification and authentication, e.g. to identify adulteration. The simplicity of layer formation via in situ photoinitiated polymerization represents the great advantage for the application of flat monoliths as TLC support. Additionally, hydrophobic/hydrophilic properties and chemical reactivity of polymer material can be easily tuned by variation of functional monomer(s) and crosslinkers.

\section{Conflicts of interest}

The authors declare no conflict of interest.

\section{Acknowledgements}

The authors would like to thank Dr. Arko Wicaksono for the Curcuma samples.

\section{References}

1 F. Svec and Y. Q. Lv, Anal. Chem., 2015, 87, 250-273.

2 S. Gocan, J. Chromatogr. Sci., 2002, 40, 538-549.

3 F. Svec and J. M. J. Frechet, Science, 1996, 273, 205-211. 
4 M. H. Mohamed and L. D. Wilson, Nanomaterials, 2012, 2, 163-186.

5 S. Mane, Can. Chem. Trans., 2016, 4, 210-225.

6 F. R. Mansour, S. Waheed, B. Paull and F. Maya, J. Sep. Sci., 2020, 43, 56-69.

7 J. F. Wang, X. Y. Jiang, H. Zhang, S. Liu, L. G. Bai and H. Y. Liu, Anal. Methods, 2015, 7, 7879-7888.

8 K. Booker, C. I. Holdsworth, C. M. Doherty, A. J. Hill, M. C. Bowyer and A. McCluskey, Org. Biomol. Chem., 2014, 12, 7201-7210.

9 R. Chen, H. Zhou, M. Liu, H. Yan and X. Qiao, TrAC, Trends Anal. Chem., 2019, 111, 1-12.

10 S. Harrisson, S. R. Mackenzie and D. M. Haddleton, Chem. Commun., 2002, 2850-2851, DOI: 10.1039/ b209479g.

11 R. Bakry, G. K. Bonn, D. Mair and F. Svec, Anal. Chem., 2007, 79, 486-493.

12 Y. Q. Lv, Z. X. Lin, T. W. Tan and F. Svec, J. Chromatogr. A, 2013, 1316, 154-159.

13 E. F. Maksimova, E. G. Vlakh and T. B. Tennikova, J. Chromatogr. A, 2011, 1218, 2425-2431.

14 D. Yin, Y. Guan, H. Gu, Y. Jia and Q. zhang, RSC Adv., 2017, 7, 7303-7309.

15 E. Korzhikova-Vlakh, M. Antipchik and T. Tennikova, Polymers, 2021, 13, 1059.
16 M. Hummel, B. Naier, H. Schottenberger, H. Huppertz, G. Partl and M. Noisterning, Composition comprising a fluorine-containing surfactant, WO2017144181A1, 2017.

17 M. Hummel, M. Markiewicz, S. Stolte, M. Noisternig, D. E. Braun, T. Gelbrich, U. J. Griesser, G. Partl, B. Naier, K. Wurst, B. Kruger, H. Kopacka, G. Laus, H. Huppertz and H. Schottenberger, Green Chem., 2017, 19, 3225-3237.

18 T. Kowalska, K. Kaczmarski and W. Prus, Handbook of ThinLayer Chromatography, 2003, 2, 47-80.

19 S. Z. Yu, F. L. Ng, K. C. C. Ma, A. A. Mon, F. L. Ng and Y. Y. Ng, J. Appl. Polym. Sci., 2013, 127, 2641-2647.

20 J. C. Giddings, G. H. Stewart and A. L. Ruoff, J. Chromatogr., 1960, 3, 239-251.

21 B. Spangenberg, C. F. Poole and C. Weins, Quantitative Thin-Layer Chromatography, Springer Berlin Heidelberg, Berlin, Heidelberg, 2011.

22 G. Guiochon and A. Siouffi, J. Chromatogr. Sci., 1978, 16, 598-609.

23 B. Fried and B. Sherma, Thin-Layer Chromatography, revised and expanded, CRC Press, 1999.

24 R. Borisov, A. Kanateva and D. Zhilyaev, Front. Chem., 2021, 9.

25 T. Wikara, A. Sulistiowaty, S. Murhandini and T. Usia, Jurnal Jamu Indonesia, 2016, 1, 9-14.

26 A. Rohman, T. Wijayanti, A. Windarsih and S. Riyanto, Molecules, 2020, 25, 3928. 